\title{
Low-cost wireless mesh communications based on openWRT and voice over internet protocol
}

\author{
Nina Siti Aminah, Muhamamad Reza Ramadhani Raharjo, Maman Budiman \\ IoT Laboratory, Instrumentation and Computational Physics, Institut Teknologi Bandung, Indonesia
}

\begin{tabular}{|c|c|}
\hline Article Info & ABSTRACT \\
\hline Article history: & Technology makes it easier for us to communicate over a distance. However, \\
\hline Received Nov 2, 2020 & $\begin{array}{l}\text { there are still many remote areas that find it difficult to communicate. This is } \\
\text { due to the fact that communication infrastructure in some areas is expensive }\end{array}$ \\
\hline Revised Apr 21, 2021 & to build while the profit will be low. This paper proposes to combine voice \\
\hline Accepted May 17, 2021 & $\begin{array}{l}\text { over internet protocol (VoIP) over mesh network implemented on openWRT } \\
\text { router. The routers are performing mesh functions. We set up a VoIP server }\end{array}$ \\
\hline Keywords: & $\begin{array}{l}\text { on a router and enabled session initiation protocol (SIP) clients on other } \\
\text { routers. Therefore, we only need routers as a means of communication. The }\end{array}$ \\
\hline $\begin{array}{l}\text { Mesh networks } \\
\text { Network topology } \\
\text { OpenWRT }\end{array}$ & $\begin{array}{l}\text { experiment showed very good results, in the line-of-sight (LOS) condition, } \\
\text { they are limited to reception distances up to } 145 \text { meters while in the non-line- } \\
\text { of-sight (NLOS) condition, they are limited to reception distances up to } \\
55 \text { meters. }\end{array}$ \\
\hline
\end{tabular}

This is an open access article under the CC BY-SA license.

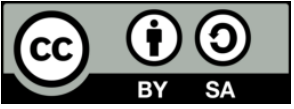

\section{Corresponding Author:}

Nina Siti Aminah

Instrumentation and Computational Physics

Institut Teknologi Bandung

Ganesha 10, Bandung 40132, West Java, Indonesia

Email: nina@fi.itb.ac.id

\section{INTRODUCTION}

One important aspect of the development of communication technology is the existence of wireless technology. Voice over internet protocol (VoIP) is one of the wireless technologies that can help people communicate. VoIP in general is a system that utilizes internet protocol to be able to transmit data in the form of voice through wireless signals [1]-[3]. The application of VoIP using wireless found many problems, one of which was the ability of the router to be inflexible and still need an access point in its network topology. Faced with this problem, developers from Germany who called themselves freifunk created a network system in the form of "Mesh", which is a method that can heal its own network independently [4]. In addition, this system can also adapt to changes in the infrastructure nodes and can use multi-hop between one nodes with another node. This mesh network and VoIP solution certainly helps a lot if implemented in difficult terrain areas such as in remote areas to conduct search and rescue, battlefield communication, or communication to help victims of natural disasters that require high mobility. This solution is also expected to help villages that cannot be reached by technology, for example in remote areas, where communication infrastructure is not necessarily available or economically feasible. Indonesian Ministry of Communication and Information Technology (KOMINFO) data for 2017 shows that 40\% of regions in Indonesia do not yet have a communication infrastructure [5].

Meanwhile, mobile ad hoc network (MANET) is a wireless technology that does not require an infrastructure in the network, making it easier for users to communicate by utilizing the presence of their mobile device [6]-[8]. The telephony world has transformed packet-switching technology to carry voice 
payload over internet protocol (IP) networks. This step leads to the growing importance of VoIP. VoIP in MANET needs session initiation protocol (SIP) for signaling [9]-[12].

B.A.T.M.A.N. stands for better approach to mobile ad hoc networking, is a routing protocol for MANET. B.A.T.M.A.N. can be installed on a router that has openWRT installed so that it can use mesh networking. B.A.T.M.A.N has a better performance than optimized link state routing protocol (OLSR) regarding packet loss metrics. Packet buffering is used by B.A.T.M.A.N unstable routes. However, delay is still increasing [13]-[17].

Therefore, in this research we try to facilitate both short and long-distance communication in remote areas using VoIP combined with a mesh network. The mesh network function is to establish networks between routers independently. This research tries to create a VoIP server and SIP client on a router, so we only need a router as a means of communication. The use of a router for communication is inexpensive, a router used in this research cost only 18 United States dollar (USD).

\section{RESEARCH METHOD}

The research begins with designing an operating system that can be used on a TP-LINK MR3020 router. The design of the operating system consists of selecting the packages needed and eliminating unnecessary packages. The required packages, namely B.A.T.M.A.N, Asterisk, batctl, baresip, and ASIAN Law Students' Association (ALSA) to create a stable operating system and minimum storage usage. Next step is to install operating system. OpenWRT is used as the operating system. OpenWRT is a LINUX-based operating system (OS) that can replace the default OS of a router. The function of OpenWRT is to facilitate users in order to maximize the router utilization. The unique feature of OpenWRT is that it is a writeable root file system, users can change the root of the router system, modify any file, and easily install software [18], [19]. OpenWRT has used in communication projects in remote areas, namely Village Telco project [20], Wray project in England [21], AirJaldi network in India [22], Pebbles valley mesh network in South Africa [23], and Tegola mesh [24], [25].

There are two router systems used in this research, the first one is the router system that is used as a server, the router will be installed with an asterisk as a telephone control center, here we can register the phone number we want so that it can be used by the SIP clients later. The second router system is an SIP client, this router is used to call one router using another router. This router will have a universal serial bus (USB) soundcard that is used for sound input and sound output. All installed routers will be integrated with mesh network. The relationship between the server and all stateful packet inspection (SPI) clients can be seen in an overview in Figure 1.

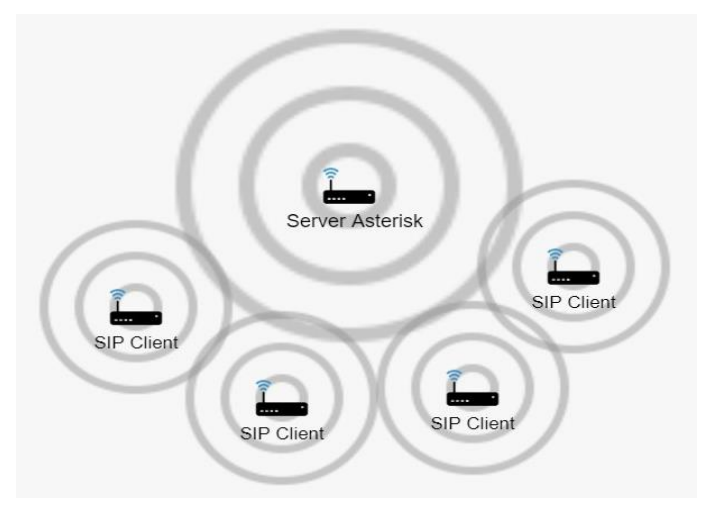

Figure 1. System overview

The first step to use a router as a server is compiling openWRT firmware which will be installed on a router. Compiling the OpenWRT firmware must be done on a computer with a Linux operating system installed. In this research, the authors use Ubuntu 18.04.3 long term service (LTS). To design OpenWRT, we must determine what packages are needed in this research. For the server router we used asterisk15, batman$\mathrm{adv}$, batctl, asterisk15-chan-sip, asterisk15-res-rtp-asterisk and luci. Asterisk15 is the main package of the VoIP server that we want to build, while asterisk15-chan-sip and asterisk15-res-rtp-asterisk are the modules that must be installed for the VoIP server to work. Batman-adv is the main package for building a mesh network, while batctl is a package that can configure batman-adv. 
Basically, compiling the OpenWRT firmware for SIP clients is the same as compiling the openWRT firmware for the server, the difference is the packages that are filled in the firmware, the required packages are batman-adv, batctl, luci, baresip, baresip baresip-mod-alsa, baresip-mod-cons, baresip-mod-evdev, baresip-mod-g711, baresip-mod-stdio, baresip-mod-uuid, alsa-utils, alsa-libs, kmod-sound-core, kmod-usbaudio, kmod-usb-hid, kmod-hid, kmod-hid-generic. Baresip is a modular SIP client, baresip itself requires several modules such as mod alsa so that baresip can work with alsa, mod g711, so that audio codec can be used and so on. Alsa-utils is a package from ALSA to run voice commands, while alsa-lib is a library of ALSA itself. Kmod-sound-core and kmod-usb-audio are the kernel modules used so that router can accept input from the USB Soundcard. Kmod-usb-hid, kmod-hid and kmod-hid-generic are the kernel modules used so that router can use a USB keypad.

After openWRT has successfully installed, the next step is to configure router so that it can recognize other routers and the router can be used as a server or SIP clients. The configuration carried out on server, namely phone number registration, password, and what the server does when it gets an incoming call in the form of an invite. Meanwhile, the configuration carried out on the SIP client, namely phone number registration that will be used by the SIP client and sound driver configuration. In this research the authors use the ALSA driver. A flowchart representing all process can be seen in Figure 2.

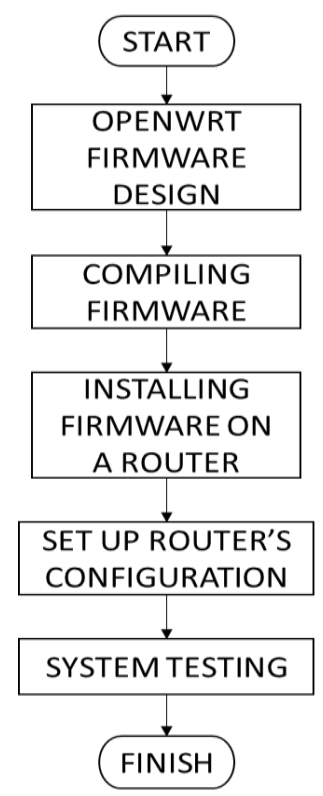

Figure 2. Research flowchart

\section{RESULTS AND DISCUSSION}

The prototype of the router can is shown in Figure 3. Server router controls incoming calls and outgoing calls, this router decides whether the incoming calls are in the network system or not. The problem with the MR3020 router is its small capacity so that additional memory is needed in the form of a flash disk. The flash disk that has been formatted into ext4 and linux swap format functions as additional random access memory (RAM) so the routers can do optimal work. The SIP client prototype is the same as the router server, it only has a few additions. The first is the additional memory in the form of a flash disk. Second, SIP client requires a USB soundcard to support audio input and output. The USB soundcard connects to a microphone as audio input, and a speaker or headset as audio output. The third is the USB keypad, this keypad functions as an input command for router such as picking up a phone call, inputting the number, and hanging up a phone call.

Tests are carried out to determine the effect of a hop on the mesh network performance. The test conducted by placing nodes with a certain distance in condition of no obstacles such as walls, trees, this condition is called line of sight (LOS). LOS testing is carried out in II street, Institut Teknologi Bandung. This can be seen from Figure 4(a). Then the next condition is to put nodes with a certain distance across a path that is partially obstructed by obstacles, this condition is called non line of sight (NLOS). NLOS testing is carried out in physics building, Institut Teknologi Bandung. This can be seen from Figure 4(b). Both tests are carried out in the morning from nine to eleven. 


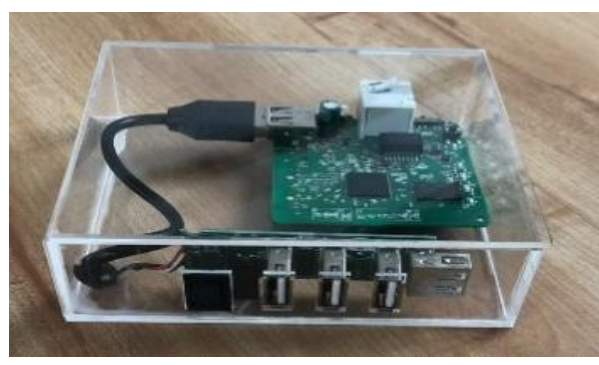

Figure 3. Prototype of the router

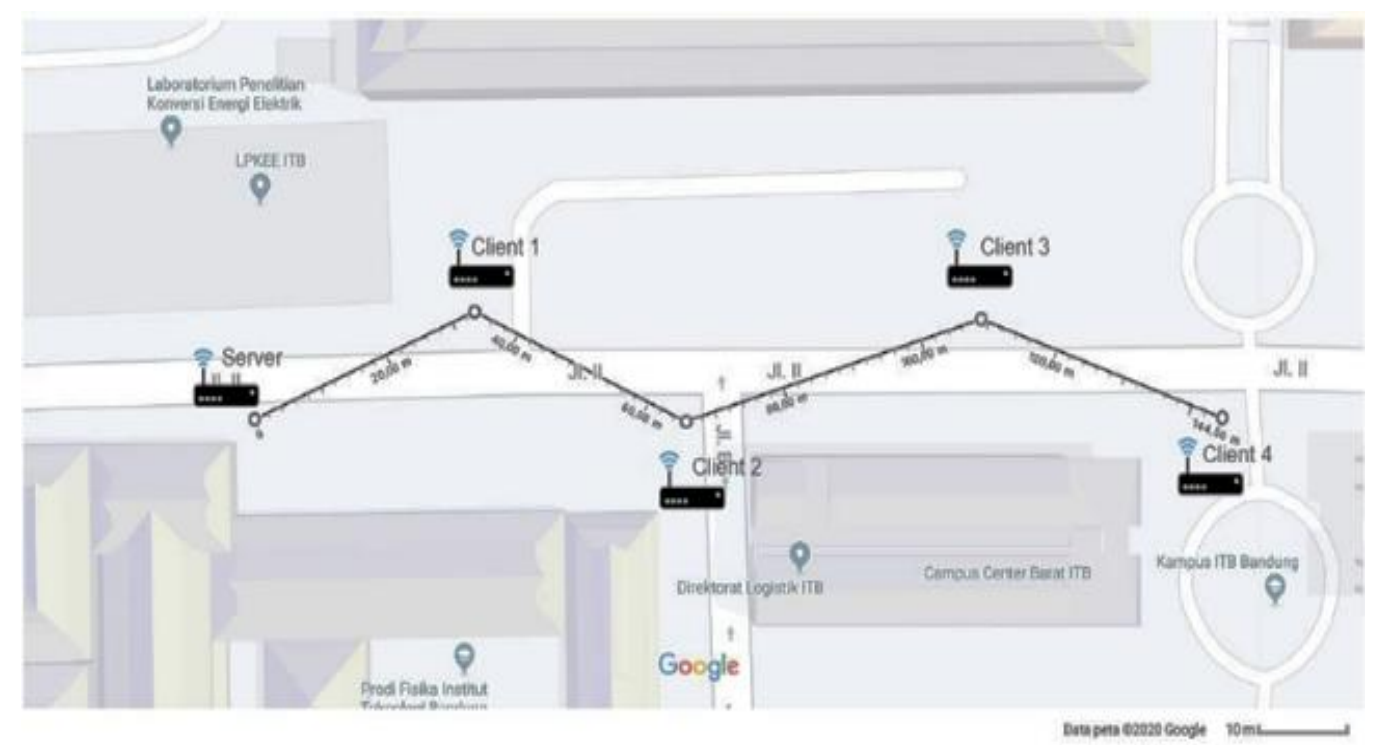

(a)

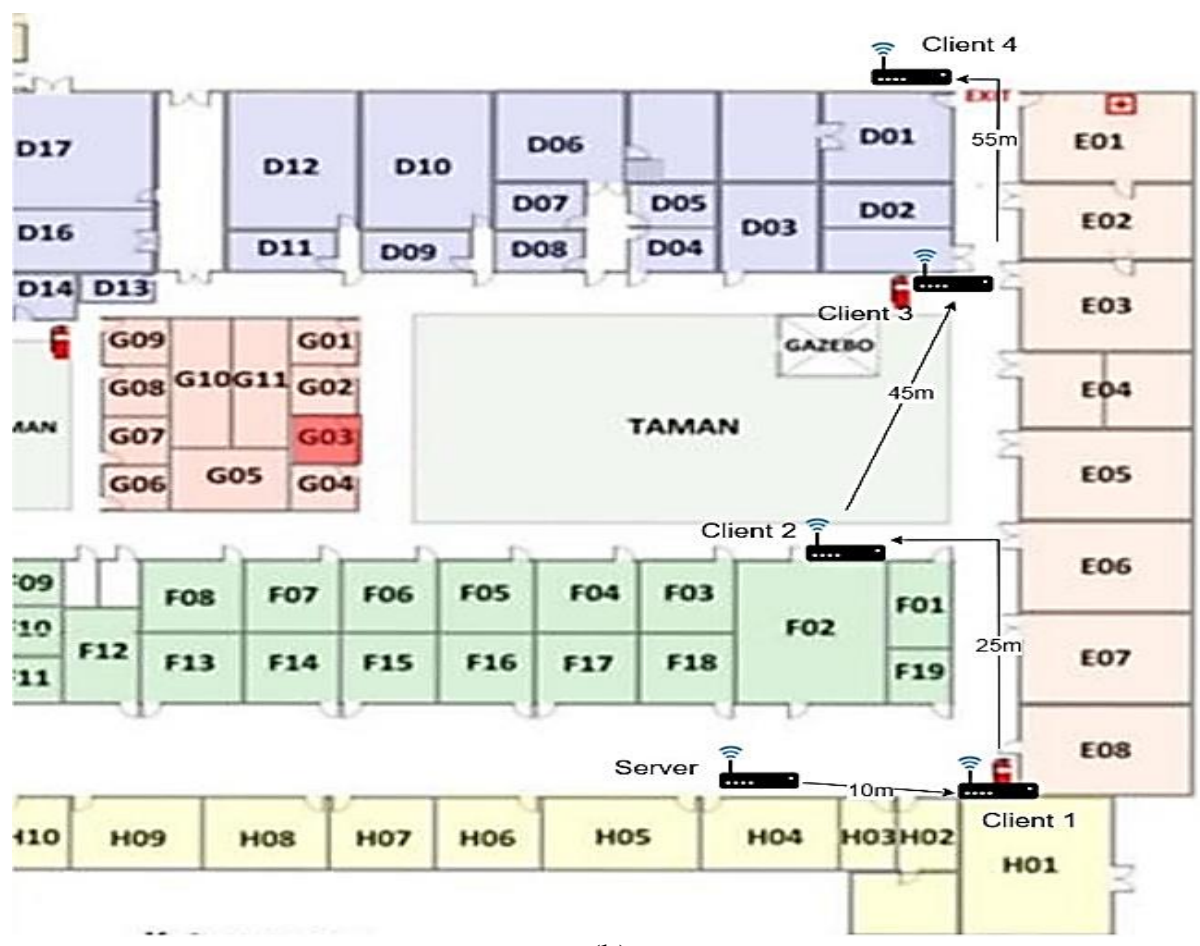

(b)

Figure 4. Multi-hop testing, (a) in LOS condition, (b) in NLOS condition 
The parameters tested are throughput, download rate, ping time and packet loss. Throughput is the number of bytes that can be sent at certain interval, in this study the authors set ten seconds interval, from the throughput data we will get the download rate data in unit kilobits per second (Kbps). Throughput and download rate tests were carried out five times and the average value was calculated. Ping time is router response times. When a router wants to calculate the ping time from router A to router $\mathrm{B}$, router $\mathrm{A}$ will send a packet and router B will respond by sending a packet back, ping time shows how fast this process happened. While packet loss is the packets that fail to arrive at their destination.

In this study, the authors measured throughput and download rate using stationary router with multi-hop scheme for maximum four hops. Throughput and download rate data showed router performance regarding their ability to send data from one node to another. Figures 5 and 6 shows that an increase in hops caused a decrease in the value of the throughput, this means that the number of hops affects the router performance in sending information. In the first hop, for LOS, the throughput value is 8.648.474, while the download rate is $6666 \mathrm{Kbps}$. In the second hop the value decreases to 2.038.356, while the download rate is $1584 \mathrm{Kbps}$. The value of throughput will affect the amount of traffic that can pass through the router, in VoIP server, it determines how many calls can occur. The first and second hop consider capable of making 10 calls simultaneously, but third and fourth hop only able to make 3 calls simultaneously. NLOS data can be seen in Figure 5, in the first hop, the throughput value is 18.212 .659 , while in the third hop the throughput value decreases to 2.081.750. The graph shows decreasing throughput and download rate value in line with the number of hops. From the data above, the NLOS conditions are better than the LOS conditions. But the data is taken at the different distance between routers. The maximum distance between routers that could be tolerated in NLOS conditions was only $55 \mathrm{~m}$ while the maximum distance between routers that could be tolerated in LOS conditions was $145 \mathrm{~m}$. This was a good result when compared to a similar reference data, where the maximum horizontal distance between routers that could be tolerated in NLOS conditions was $40 \mathrm{~m}$ while the maximum horizontal distance between routers that could be tolerated in LOS conditions was $52 \mathrm{~m}[26]$.

Throughput Data

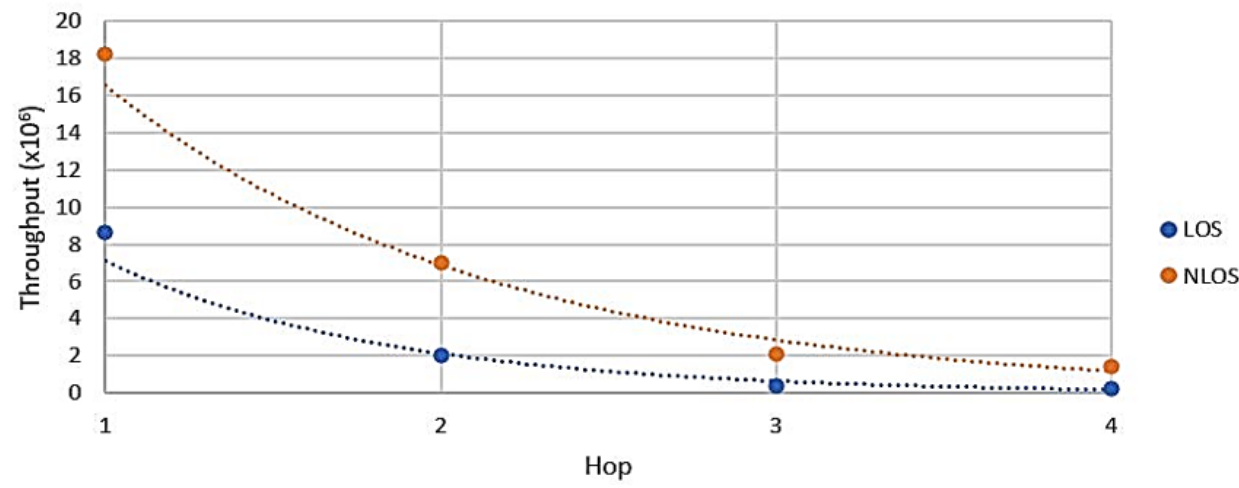

Figure 5. Throughput data

Download Rate Data

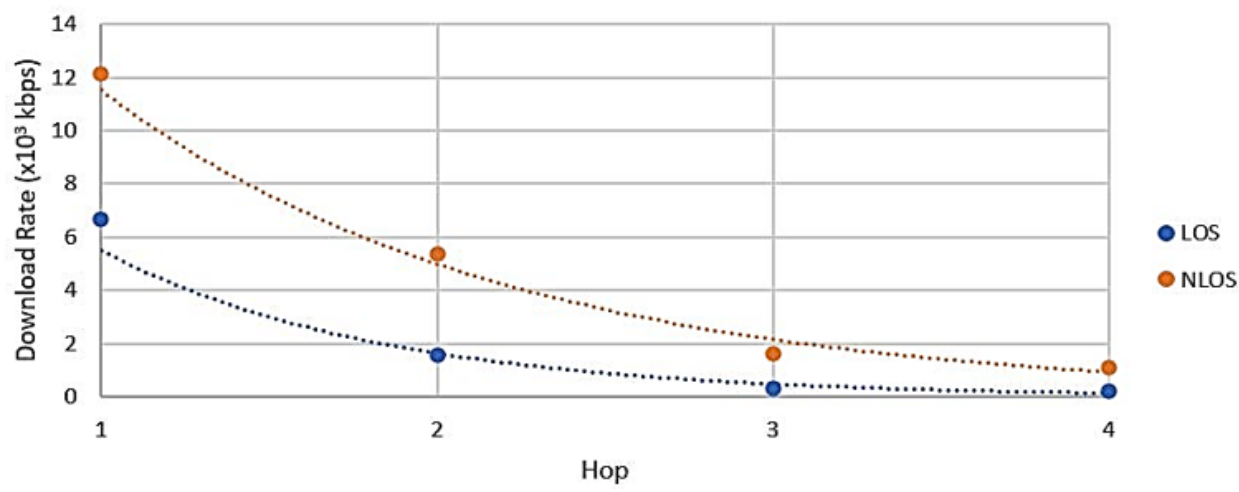

Figure 6. Download rate data 
All graphs obtained have similar shape to the reference [26]. Improving the throughput data, can be done by adding an external antenna to the router. Antenna will help router to send and receive signals better, or simply replace the router used with a router that has a better antenna reception. Ping time is time needed to send and receive a response from one router to another router. In this study, the authors sent 120 packets data from the server router to client. It can be seen from Figure 7 that in the first hop, the ping time value is $7 \mathrm{~ms}$, then in the third hop the ping time value rises to $16 \mathrm{~ms}$ and reachest $31 \mathrm{~ms}$ in the fourth hop. The more hops, the longer time needed for the packet to be received. A high ping time value produced a pause voice during a call. The NLOS ping time data is no more than $7 \mathrm{~ms}$, which can be seen in Figure 7. This is due to the close distance between each router in NLOS conditions.

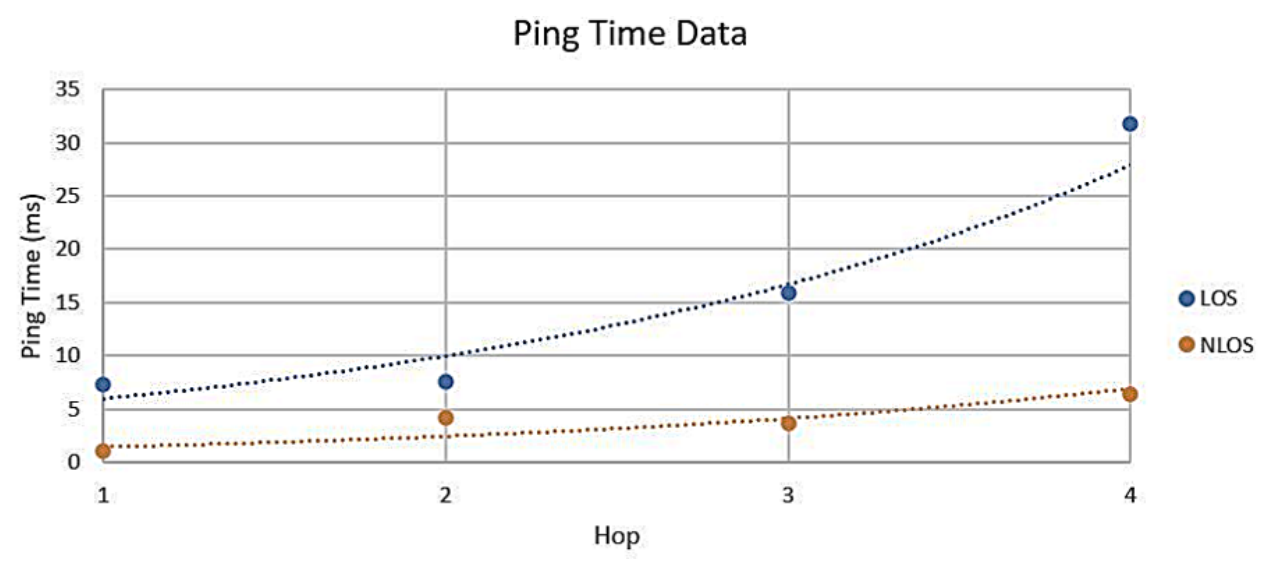

Figure 7. Ping time data

Packet loss is the packets that fail to arrive at their destination. There are 120 packets sent. It can be seen from Figure 8 that more hops generate more packets fail to reach their destination. In the first hop, the LOS packets failed no more than $1 \%$, but in the fourth hop there are 20 packets or $16 \%$ of the total packages failed to arrive at their destination. This generated an intermittent issue during calls. The packet loss data in NLOS conditions is no more than $2 \%$, this is due to the close distance between each router in NLOS conditions.

\section{Packet Loss Data}

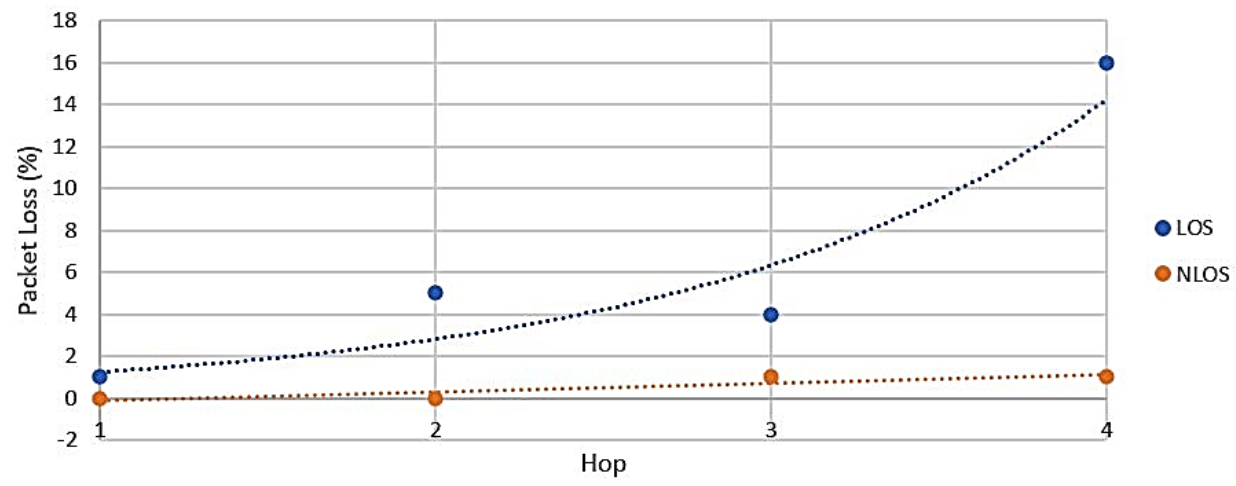

Figure 8. Packet loss data

\section{CONCLUSION}

In this research, short and long-distance communication in remote areas using (VoIP) combined with a mesh network is implemented. In this research, we set up a VoIP server along with an SIP client on a router, therefore only needed a router as a means of communication. Several parameters measured to test its performance, namely throughput, download rate, ping time and packet loss. The highest throughput value is 
8.648.474 under LOS conditions and 18.212.659 under NLOS conditions. The highest ping time is $32 \mathrm{~ms}$ under LOS conditions and $7 \mathrm{~ms}$ under NLOS conditions. The highest packet loss is $16 \%$ under LOS conditions and $1 \%$ under NLOS conditions. Wall affects the performance of the router, it can be seen that in the LOS condition, the total distance between each router is 145 meters while in the NLOS condition, the total distance between each router is only 55 meters. Router can be used as a VoIP server and SIP client. The SIP client prototype is the same as the router server, it only has a few additions, namely a flash disk, a USB Soundcard connected to a microphone a speaker or headset, and a USB keypad.

\section{ACKNOWLEDGEMENTS}

The authors would like to acknowledge the International Research Grant, World Class University, Institut Teknologi Bandung for funding this research.

\section{REFERENCES}

[1] L. Sun, I. H. Mkwawa, E. Jammeh, and E. Ifeachor, "Guide to Voice and Video over IP: for fixed and mobile networks," London: Springer-Verlag, 2013.

[2] B. Goode, "Voice over Internet protocol (VoIP)," in Proceedings of the IEEE, vol. 90, no. 9, pp. 1495-1517, Sept. 2002, doi: 10.1109/JPROC.2002.802005.

[3] G. F. Mao, A. Talevski, and E. Chang, "Voice over Internet Protocol on Mobile Devices," in 6th IEEE/ACIS International Conference on Computer and Information Science (ICIS 2007), Jul. 2007, pp. 163-169, doi: 10.1109/ICIS.2007.131

[4] T. Hardes, F. Dressler, and C. Sommer, "Simulating a city-scale community network: From models to first improvements for Freifunk," 2017 International Conference on Networked Systems (NetSys), 2017, pp. 1-7, doi: 10.1109/NetSys.2017.7903954.

[5] A. N. Afidah, M. Doom, and R. N. S. Putri, “2017 Performance Report,” (Indonesian Language), Indonesian Ministry of Communication and Information Technology, Jakarta, Indonesia, March 2018.

[6] N. Muthukumaran, "Analyzing Throughput of MANET with Reduced Packet Loss," Wireless Personal Communications, vol. 97, no. 1, pp. 565-578, Nov. 2017, doi: 10.1007/s11277-017-4520-9.

[7] J. Swain, B. K. Pattanayak, and B. Pati, "Study and analysis of routing issues in MANET," 2017 International Conference on Inventive Communication and Computational Technologies (ICICCT), 2017, pp. 506-509, doi: 10.1109/ICICCT.2017.7975251.

[8] G. Ravi and A. S. Saranya, "Optimized power management for multi hop mobile adhoc networks," 2015 International Conference on Communications and Signal Processing (ICCSP), 2015, pp. 1165-1170, doi: 10.1109/ICCSP.2015.7322688.

[9] L. A. Haibeh, N. Hakem, and O. A. Safia, "Performance evaluation of VoIP calls over MANET for different voice codecs," in 2017 IEEE 7th Annual Computing and Communication Workshop and Conference (CCWC), Jan. 2017, pp. 1-6, doi: 10.1109/CCWC.2017.7868479.

[10] N. K. Patel, P. Saxena, R. Singh, and S. Kumar, "A Novel Voice Signaling Protocol for Tactical Broadcast Voice Communication in Mobile Ad Hoc Network," 2019 10th International Conference on Computing, Communication and Networking Technologies (ICCCNT), 2019, pp. 1-4, doi: 10.1109/ICCCNT45670.2019.8944860.

[11] M. Alshamrani, "SIP over Next Generation mobile ad hoc networks (MANETs)," 2013 IEEE 14th International Symposium on "A World of Wireless, Mobile and Multimedia Networks" (WoWMoM), 2013, pp. 1-2, doi: 10.1109/WoWMoM.2013.6583433.

[12] M. Alshamrani, H. Cruickshank, Z. Sun, V. Fami, and B. Elmasri, "Evaluation of SIP Signalling and QoS for VoIP over MANETs Reactive Routing Protocols," 2013 Seventh International Conference on Next Generation Mobile Apps, Services and Technologies, 2013, pp. 105-110, doi: 10.1109/NGMAST.2013.27.

[13] E. Kulla, M. Hiyama, M. Ikeda, and L Barolli, "Performance comparison of OLSR and BATMAN routing protocols by a MANET testbed in stairs environment," Computers and Mathematics with Applications, vol. 62, no. 2, pp. 339-349, Jan. 2012, doi: 10.1016/j.camwa.2011.07.035.

[14] L. Barolli, M. Ikeda, G. De Marco, A. Durresi, and F. Xhafa, "Performance Analysis of OLSR and BATMAN Protocols Considering Link Quality Parameter," 2009 International Conference on Advanced Information Networking and Applications, 2009, pp. 307-314, doi: 10.1109/AINA.2009.28.

[15] P. Vilhan and L. Hudec, "Building Public Key Infrastructure for MANET with Help of B.A.T.M.A.N. Advanced," 2013 European Modelling Symposium, 2013, pp. 566-571, doi: 10.1109/EMS.2013.94.

[16] M. Britton and A. Coyle, "Performance analysis of the B.A.T.M.A.N. wireless ad-hoc network routing protocol with mobility and directional antennas," 2011 Military Communications and Information Systems Conference, 2011, pp. 1-6, doi: 10.1109/MilCIS.2011.6470393.

[17] R. Botta et al., "Investigation of silver nanorods as reusable SERS-active substrates for trace level detection of 2MIB volatile organic compound," Sensors and Actuators B: Chemical, vol. 271, pp. 122-127, Oct. 2018, doi: 10.1016/j.snb.2018.05.110.

[18] C. E. Palazzi, M. Brunati, and M. Roccetti, "An OpenWRT solution for future wireless homes," 2010 IEEE International Conference on Multimedia and Expo, 2010, pp. 1701-1706, doi: 10.1109/ICME.2010.5583223. 
[19] M. Kciuk, "OpenWRT operating system based controllers for mobile robot and building automation system students projects realization," 15th International Workshop on Research and Education in Mechatronics (REM), 2014, pp. 1-4, doi: 10.1109/REM.2014.6920248.

[20] M. Adeyeye and P. Gardner-Stephen, "The Village Telco project: a reliable and practical wireless mesh telephony infrastructure," EURASIP Journal on Wireless Communications and Networking, vol. 2011, no. 1, pp. 1-11, Aug. 2011, doi: 10.1186/1687-1499-2011-78.

[21] J. Ishmael, S. Bury, D. Pezaros, and N. Race, "Deploying Rural Community Wireless Mesh Networks," in IEEE Internet Computing, vol. 12, no. 4, pp. 22-29, July-Aug. 2008, doi: 10.1109/MIC.2008.76.

[22] S. Surana, R. K. Patra, S. Nedevschi, M. Ramos, L. Subramanian, and E. Brewer, "Beyond pilots: Keeping rural wireless networks alive," ACMUSENIX Networked Syst. Des. Implement. NSDI, vol. 8, pp. 119-132, 2008, Accessed: Oct. 31, 2020. [Online]. Available: https://nyuscholars.nyu.edu/en/publications/beyond-pilots-keepingrural-wireless-networks-alive.

[23] D. L. Johnson and K. Roux, "Building rural wireless networks: lessons learnt and future directions," in Proceedings of the 2008 ACM workshop on Wireless networks and systems for developing regions, New York, NY, USA, Sep. 2008, pp. 17-22, doi: 10.1145/1410064.1410068.

[24] Asriadi, I. Pratomo, A. Affandi, and D. S. Rahardjo, "OpenVoice: Low-cost mobile wireless communication project for rural area based on OpenWRT," 2015 International Seminar on Intelligent Technology and Its Applications (ISITIA), 2015, pp. 391-396, doi: 10.1109/ISITIA.2015.7220012.

[25] G. Bernardi, P. Buneman, and M. K. Marina, "Tegola tiered mesh network testbed in rural Scotland," in Proceedings of the 2008 ACM workshop on Wireless networks and systems for developing regions, New York, NY, USA, Sep. 2008, pp. 9-16, doi: 10.1145/1410064.1410067.

[26] M. Hariyadi, I. Pratomo and A. Affandi, "Implementation and performance analysis of routing BATMAN on VoIP applications in the campus area," 2016 International Seminar on Intelligent Technology and Its Applications (ISITIA), 2016, pp. 333-338, doi: 10.1109/ISITIA.2016.7828682.

\section{BIOGRAPHIES OF AUTHORS}

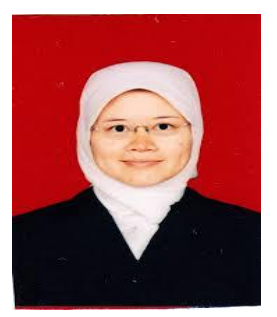

Nina Siti Aminah was born in Bogor, Indonesia. She received the B.Eng. degree in electronics from the National Institute of Technology, Indonesia, and the B.S., M.S., and Ph.D. degrees in physics from Bandung Institute of Technology, Indonesia. She is currently a postdoctoral fellow at University of Indonesia and lecturer at the Instrumentation and Computational Physics Research Group, Bandung Institute of Technology.

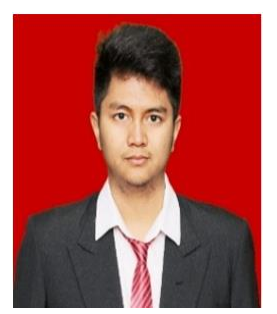

Muhamamad Reza Ramadhani Raharjo born in Bandung, 29 January 1997 is third child of three siblings. Started his formal education at SD Sultan agung (2002-2005) in Pematang Siantar, then he continued his study at SDN Banjarsari in Bandung (2005-2008). Then he went to study at SMP 7 Bandung (2008-2011). Graduated in 2014 from SMAN 5 Bandung. He decided took a gap year after graduated from high school. Started his university life in 2015 at Bandung Institute of Technology as a student of Department of Physics and got his degree in 2020 .

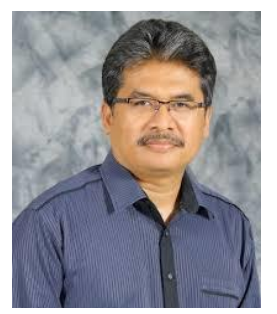

Maman Budiman was born in Garut, Indonesia. He received the B.S. degree in physics from the Bandung Institute of Technology, Indonesia, and the M.Eng. and Ph.D. degrees in electrical engineering from Tokyo Institute of Technology, Japan. He is currently a Lecturer at the Instrumentation and Computational Physics Research Group and Head of IoT Laboratory, Bandung Institute of Technology. 